Received: 1 August 2017

Accepted: 26 January 2018

Published online: 08 February 2018

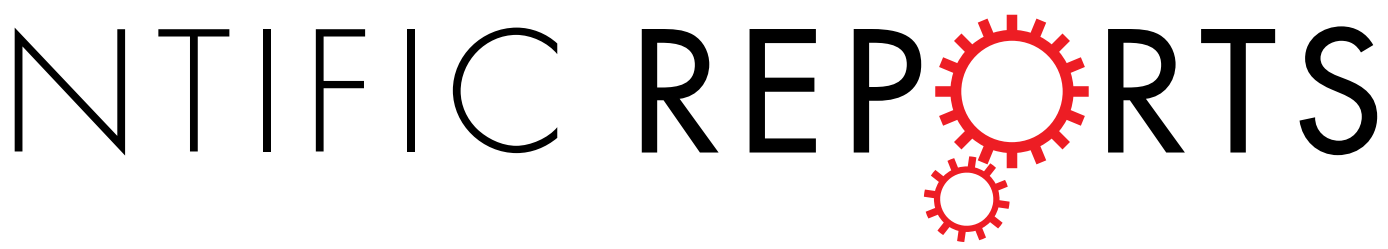

\title{
OPEN The model of circulating immune complexes and interleukin-6 improves the prediction of disease activity in systemic lupus erythematosus
}

\author{
Chokchai Thanadetsuntorn ${ }^{1}$, Pintip Ngamjanyaporn ${ }^{1}$, Chavachol Setthaudom ${ }^{2}$, \\ Kenneth Hodge ${ }^{3}$, Nisara Saengpiya ${ }^{2}$ \& Prapaporn Pisitkun ${ }^{1}$
}

Systemic Lupus Erythematosus (SLE) is an autoimmune disease resulting in autoantibody production, immune complex deposition, and complement activation. The standard biomarkers such as anti-dsDNA and complements ( $\mathrm{C} 3$ and $\mathrm{C4}$ ) do not always correlate with active clinical SLE. The heterogeneity of SLE patients may require additional biomarkers to designate disease activity. Ninety SLE patients participated in this study. Evaluation of disease activity was achieved with the Systemic Lupus Erythematosus Disease Activity Index 2000 (SLEDAI-2K) and modified SLEDAI-2K. The measured serum biomarkers were anti-dsDNA, C3, C4, ESR, interleukin-6 (IL-6), and circulating immune complexes (CIC). IL-6, ESR and CIC significantly increased in active clinical SLE. Complement, anti-dsDNA, ESR and CIC correlated with SLEDAI-2K while only anti-dsDNA, CIC, ESR and IL- 6 correlated with modified SLEDAI-2K. A combination of biomarkers gave a higher odds ratio (OR) than any single biomarker. A combination of IL-6 or CIC exhibited the highest OR (OR $=7.27,95 \% \mathrm{Cl}(1.99-26.63), \mathrm{p}=0.003)$ while either complement or anti-dsDNA showed a moderate odds ratio $(\mathrm{OR}=3.14,95 \% \mathrm{Cl}(1.16-8.48)$, $\mathrm{p}=0.024$ ) of predicting clinical active SLE. The combination of CIC and IL- 6 strongly predicts active clinical SLE. CIC and IL- 6 can be used in addition to standard biomarkers to determine SLE activity.

Systemic Lupus Erythematosus (SLE) is an autoimmune disease affecting all organ systems leading to inflammation and tissue damage ${ }^{1}$. The classical immunological abnormalities found in SLE are autoantibody production, immune complex deposition and complement activation ${ }^{2}$. The pathogenesis of SLE is complex and involves several genetic abnormalities which result in heterogeneity in disease manifestations ${ }^{3}$. The outcomes of SLE progression in patients can be devastating, with cumulative morbidity over time from disease activity or infectious complication ${ }^{4}$. Most of the clinical trials conducted with SLE patients fail to show potency of the treatment to control disease activity, although some treatments showed efficacy in some case reports 5 . One confounding problem may be the lack of sufficiently sensitive tools to monitor SLE disease activity. Accurate assessment of SLE disease activity is necessary to help physicians differentiate active disease from chronic non-inflammatory injury and provide the patient with appropriate treatment.

Beyond genetics and environmental factors, cytokine dysregulation is ubiquitous, and their proteins and gene expression profiles may serve as markers of disease activity and severity. From previous studies, the key cytokine involved in SLE pathogenesis is interferon alpha (IFN- $\alpha$ ) which leads to upregulation of several inflammatory proteins $^{6}$. Additionally, IL-6, TNF- $\alpha$, IFN- $\gamma$, and BLyS, as well as T-cell-derived cytokines like IL-17, IL-21, and IL-2, are dysregulated in $\mathrm{SLE}^{3,7}$.

${ }^{1}$ Division of Allergy Immunology and Rheumatology, Department of Medicine, Faculty of Medicine Ramathibodi Hospital, Mahidol University, Rama VI Road, Bangkok, 10400, Thailand. ${ }^{2}$ Immunology Laboratory, Department of Pathology, Faculty of Medicine Ramathibodi Hospital, Mahidol University, Rama VI Road, Bangkok, 10400, Thailand. ${ }^{3}$ Center of Excellence in Systems Biology, Faculty of Medicine, Chulalongkorn University, Bangkok, 10330, Thailand. Correspondence and requests for materials should be addressed to P.P. (email: prapaporn.pis@mahidol.ac.th) 
IL-6 can promote activation and differentiation of cells central to the development of systemic autoimmunity and the associated pathologic inflammatory responses ${ }^{8}$. There is evidence that serum levels of IL- 6 are elevated in human SLE and have correlated with disease activity or anti-dsDNA levels in some studies ${ }^{9-11}$. However, IL-6 production in LPS stimulated whole blood culture was lower in SLE patients compared to controls in another study $^{12}$. IL-6 closely linked with specific disease manifestations of SLE patients. Urinary IL- 6 correlates with titers of anti-dsDNA antibodies and decreases following treatment in patients with lupus nephritis. Also, the expression of IL-6 increased in glomerular and tubular tissue in lupus nephritis kidneys ${ }^{13}$. On the other hand, another study showed higher levels of IL-6 in SLE with hematological manifestation, but did not correlate with other individual organ and systemic disease activity ${ }^{14}$.

Elevated serum levels of circulating immune complexes (CIC) have long been described in lupus, which leads to organ inflammation and damage by immune complex deposition. Immune complexes are composed of circulating DNA and antibodies to DNA ${ }^{15}$. Few studies show the detection of CIC is specific for SLE, and correlates with disease activity and deposits in the kidney of lupus nephritis patients ${ }^{16,17}$. Several methods can be used to detect CIC, but no single procedure appears to detect all types of CICs ${ }^{18}$. Those procedures which detect CIC's containing fragments of complement (e.g. C1q and C3d) seem to match with clinically relevant events ${ }^{19}$.

There exists conflicting data on the correlation between SLE disease activity with serum IL- 6 and CIC, and those studies have been performed in small groups of patients, while others are retrospective studies ${ }^{9-11,17,18}$. This study aimed to investigate whether serum levels of IL-6 and CIC correlated with SLE disease activity achieved by the Systemic Lupus Erythematosus Disease Activity Index (SLEDAI-2K) and modified SLEDAI-2K. If so, the analysis of biomarker models would be compared to reveal the model that provides the best prediction for SLE disease activity.

\section{Results}

Patient demographics and clinical characteristics. Ninety SLE patients were enrolled in the study. Active disease was defined if the clinical SLEDAI scores were greater than one. Of the 90 total patients, 27 cases (30\%) had active clinical SLE whereas 63 cases $(70 \%)$ had inactive clinical SLE. The majority of patients were female (93.3\%), and median disease duration was 83.5 months. No statistically significant difference was observed regarding gender, disease duration and underlying diseases between the active and inactive group, except the age of active SLE group was significantly lower than the inactive SLE group ( $32.67 \pm 13.34$ years versus $43.35 \pm 14.24$ years, $p=0.001$ ). The active SLE group had a statistically significant higher erythrocyte sedimentation rate (ESR) and urine protein to creatinine ratio, but lower absolute lymphocyte counts compared with patients in the inactive SLE group. Also, the active SLE group had a statistically significant higher number of patients receiving prednisolone, mycophenolate mofetil and tacrolimus than the inactive group (Table 1). The prevalence of activity of all 24 descriptors of SLEDAI-2K for the studied cohort is shown (Supplementary Table S1).

Serum biomarkers in the different stages of SLE disease activity. The serum level of C3 had a tendency to be reduced, and anti-dsDNA was slightly higher in the active clinical SLE. However, the levels of these biomarkers in both active and inactive clinical SLE did not show a statistically significant difference (Table 2). In contrast, the active clinical SLE patients showed significantly elevated levels of IL- 6 and CIC compared with the inactive ones (Table 2). The percentage of ds-DNA antibodies and complements in the SLE cohort is shown (Supplementary Table S2).

Correlation between serum biomarkers with SLE disease activity. The correlation of biomarkers with the modified SLEDAI-2K and the SLEDAI-2K is shown (Table 3). The erythrocyte sedimentation rate (ESR), IL-6, CIC, and anti-dsDNA showed a significant positive correlation with the modified SLEDAI-2K $(\mathrm{R}=0.551$, $\mathrm{p}<0.001 ; \mathrm{R}=0.313, \mathrm{p}=0.003 ; \mathrm{R}=0.331, \mathrm{p}=0.001$; and $\mathrm{R}=0.211, \mathrm{p}=0.046$, respectively), while both $\mathrm{C} 3$ and C4 did not show significant correlation (Table 3). On the other hand, IL-6 did not reveal the correlation with the SLEDAI-2K while the other biomarkers (CIC, anti-dsDNA, C3, C4, and ESR) correlated with the SLEDAI-2K $(\mathrm{R}=0.473, \mathrm{p}<0.001 ; \mathrm{R}=0.602, \mathrm{p}<0.001 ; \mathrm{R}=-0.577, \mathrm{p}<0.001 ; \mathrm{R}=-0.494, \mathrm{p}<0.001$, and $\mathrm{R}=0.468$, $\mathrm{p}<0.001$, respectively) (Table 3).

Correlation of Individual Biomarkers. Levels of IL-6 did not display significant correlation with other biomarkers (CIC, C3, C4, and anti-dsDNA) except ESR $(\mathrm{R}=0.444, \mathrm{p}<0.001)$ (Table 4). However, CIC significantly correlated with $\mathrm{C} 3(\mathrm{R}=-0.396, \mathrm{p}<0.001), \mathrm{C} 4(\mathrm{R}=-0.457, \mathrm{p}<0.001)$, and anti-dsDNA $(\mathrm{R}=0.484$, $\mathrm{p}<0.001$ ) (Table 4)

Distinction of active clinical SLE using various biomarkers. The area under the curve (AUC) of the ROC curve was analyzed to assess the performance of individual serum biomarkers to differentiate active clinical SLE. Based on the AUC of these biomarkers, CIC and IL- 6 showed better parameters for determining active SLE than anti-dsDNA, C4 and C3 (AUC $=0.698,0.677,0.634,0.410$ and 0.393 respectively). Remarkably, ESR exhibited the highest AUC $(\mathrm{AUC}=0.7028)$ in discriminating active SLE (Fig. 1). The optimal cut-off point for CIC is $4 \mathrm{RU} / \mathrm{ml}$ which gives a $70 \%$ sensitivity and $67 \%$ specificity respectively, whereas the cut-off for IL-6 is $4.35 \mathrm{pg} / \mathrm{ml}$, which gives a sensitivity of $70 \%$ and specificity of $62 \%$ respectively.

Biomarker models predict active clinical SLE. Among each biomarker tested, CIC yielded the highest sensitivity, specificity and odds ratio in predicting active clinical SLE (Table 5). The sensitivity of ESR is quite high but the specificity and OR in predicting disease flare is insignificant (Table 5). Using a combination of different biomarkers increased the sensitivity to identify active clinical SLE and reduced specificity as well (Table 5). However, the combination of CIC or IL- 6 generated a sensitivity much higher than the routine use of either low complement or anti-dsDNA ( $92.6 \%$ vs. $74.1 \%)$, while the specificity was comparable ( $47.6 \%$ vs. $52.4 \%)$ 


\begin{tabular}{|c|c|c|c|c|}
\hline Clinical parameters & Total $(\mathrm{n}=90)$ & Active SLE $(\mathbf{n}=27)$ & Inactive SLE $(n=63)$ & $p$ value \\
\hline Age, year $(\text { mean } \pm S \mathrm{SD})^{* *}$ & $40.14 \pm 14.75$ & $32.67 \pm 13.34$ & $43.35 \pm 14.24$ & 0.001 \\
\hline \multicolumn{5}{|l|}{ Gender } \\
\hline Male, n (\%) & $6(6.7)$ & $4(14.8)$ & $2(3.2)$ & 0.064 \\
\hline Female, n (\%) & $84(93.3)$ & $23(85.2)$ & $61(96.8)$ & 0.125 \\
\hline Disease duration, month (median [IQR]) & $83.5(42-132)$ & $66(21-120)$ & $84(59-132)$ & 0.25 \\
\hline ESR, mm/hr (median[IQR])* & $28.5(17-41)$ & $35(23-56)$ & $22(14-38)$ & 0.011 \\
\hline $\mathrm{WBC}$, cells $/ \mathrm{mm}^{3}($ mean $\pm \mathrm{SD})$ & $6,714 \pm 2,600$ & $7,031 \pm 2,579$ & $6,577 \pm 2,618$ & 0.818 \\
\hline $\mathrm{ALC}$, cells $/ \mathrm{mm}^{3}(\text { mean } \pm \mathrm{SD})^{*}$ & $1665 \pm 736$ & $1369 \pm 732$ & $1791 \pm 705$ & 0.014 \\
\hline Hemoglobin, g/dL (mean \pm SD) & $12.0 \pm 1.8$ & $11.4 \pm 2.4$ & $12.3 \pm 1.5$ & 0.09 \\
\hline Platelet, cells $/ \mathrm{mm}^{3}($ mean $\pm S D)$ & $232,000 \pm 77,410$ & $229,111 \pm 97,242$ & $233,523 \pm 68,048$ & 0.831 \\
\hline Serum creatinine, mg/dl (median[IQR]) & $0.71(0.63-0.88)$ & $0.68(0.63-1.17)$ & $0.72(0.63-0.86)$ & 0.250 \\
\hline UPCR (median[IQR])*** & $0.19(0.11-0.39)$ & $0.72(0.25-2.44)$ & $0.15(0.1-0.23)$ & $<0.001$ \\
\hline Hypertension, $\mathrm{n}(\%)$ & $20(22.2)$ & $4(14.81)$ & $16(25.34)$ & 0.268 \\
\hline Clinical SLEDAI (median[IQR])*** & $0(0-2)$ & $4(4-8)$ & $0(0-0)$ & $<0.001$ \\
\hline SLEDAI-2K (median[IQR])*** & $2(0-4)$ & $6(4-10)$ & $0(0-2)$ & $<0.001$ \\
\hline \multicolumn{5}{|l|}{ Treatment } \\
\hline Prednisolone usage, $\mathrm{n}(\%)^{* * *}$ & $60(66.7)$ & $27(100)$ & $33(52.4)$ & $<0.001$ \\
\hline Hydroxychloroquine usage, n (\%) & $74(82.2)$ & $20(74.1)$ & $54(85.7)$ & 0.231 \\
\hline Azathioprine usage, $\mathrm{n}(\%)$ & $31(34.4)$ & $6(22.2)$ & $25(39.7)$ & 0.11 \\
\hline Cyclophosphamide usage, n (\%) & $8(8.9)$ & $5(18.5)$ & $3(4.8)$ & 0.05 \\
\hline Mycophenolate mofetil usage, $\mathrm{n}(\%)^{*}$ & $18(20)$ & $10(37)$ & $8(12.7)$ & 0.01 \\
\hline Tacrolimus usage, $\mathrm{n}(\%)^{*}$ & $3(3.3)$ & $3(11.1)$ & $0(0)$ & 0.025 \\
\hline
\end{tabular}

Table 1. Patient demographics and clinical characteristics. ESR, erythrocyte sedimentation rate; ALC, absolute lymphocyte count; UPCR, urine protein to creatinine ratio; SLEDAI, Systemic lupus erythematosus disease activity index; $* \mathrm{p}<0.05, * * \mathrm{p}<0.01, * * * \mathrm{p}<0.001$.

\begin{tabular}{|l|l|l|l|}
\hline Biomarkers & Clinical Active $(\mathbf{n}=27)$ & Clinical Inactive $(\mathbf{n}=\mathbf{6 3})$ & $\boldsymbol{p}$-value \\
\hline $\mathrm{C} 3, \mu \mathrm{g} / \mathrm{ml}$ & $877(694-1070)$ & $1010(824-1230)$ & 0.107 \\
\hline $\mathrm{C} 4, \mu \mathrm{g} / \mathrm{ml}$ & $192(120-246)$ & $217(144-282)$ & 0.25 \\
\hline Anti-dsDNA, IU/ml & $101.4(37.2-436.5)$ & $48.9(10.0-209.8)$ & 0.25 \\
\hline $\mathrm{IL}-6, \mathrm{pg} / \mathrm{ml}{ }^{*}$ & $5.5(4.0-14.5)$ & $3.6(2.6-6.7)$ & 0.011 \\
\hline $\mathrm{CIC}, \mathrm{RU} / \mathrm{ml}^{* *}$ & $10.12(3.0-23.6)$ & $2.1(2.0-7.7)$ & 0.001 \\
\hline
\end{tabular}

Table 2. Serum biomarkers in the different stages of SLE disease activity. Data showed in median (IQR). $* \mathrm{p}<0.05, * * \mathrm{p}<0.01$.

\begin{tabular}{|l|l|l|l|l|}
\hline \multirow{2}{*}{ Serum biomarkers } & \multicolumn{2}{|l|}{ Modified SLEDAI-2K } & \multicolumn{2}{l|}{ SLEDAI-2K } \\
\cline { 2 - 5 } & Correlation coefficient & $\boldsymbol{p}$-value & Correlation coefficient & $\boldsymbol{p}$-value \\
\hline C3 & -0.203 & 0.055 & -0.577 & $<0.001^{* * *}$ \\
\hline C4 & -0.180 & 0.089 & -0.494 & $<0.001^{* * *}$ \\
\hline Anti-dsDNA & 0.211 & $0.046^{*}$ & 0.602 & $<0.001^{* * *}$ \\
\hline ESR & 0.551 & $<0.001^{* * *}$ & 0.468 & $<0.001^{* * *}$ \\
\hline IL-6 & 0.313 & $0.003^{* *}$ & 0.153 & 0.149 \\
\hline CIC & 0.331 & $0.001^{* *}$ & 0.473 & $<0.001^{* * *}$ \\
\hline
\end{tabular}

Table 3. Correlation between serum biomarkers and SLEDAI score. $* \mathrm{p}<0.05, * * \mathrm{p}<0.01, * * * \mathrm{p}<0.001$.

(Table 5). Also, the combination of IL-6 or CIC demonstrated the highest odds ratio of predicting active clinical SLE compared to the combination of complement or anti-dsDNA (OR $=7.27,95 \%$ CI $(1.99-26.63), p=0.003$ vs. $\mathrm{OR}=3.14,95 \% \mathrm{CI}(1.16-8.48), \mathrm{p}=0.024)$ (Table 5). Although ESR showed a moderate degree of correlation with IL-6 (Table 4), the combination of ESR and CIC did not show a significant OR in predicting active disease $(\mathrm{OR}=3.45,95 \% \mathrm{CI}(0.93-12.87), \mathrm{p}=0.065)$ (Table 5).

Also, seven out of twenty-seven of active clinical SLE cases showed normal levels of complement and anti-dsDNA. Up to $85 \%(6 / 7)$ had either elevated CIC or IL-6. The clinical manifestations included rash, lymphopenia, autoimmune hemolytic anemia (AIHA), psychosis, myelitis and mild lupus nephritis (Table 6). 


\begin{tabular}{|l|l|l|l|l|l|l|}
\hline Biomarkers & Statistical Analysis & C3 & C4 & Anti-dsDNA & ESR & CIC \\
\hline \multirow{2}{*}{ IL-6 } & Correlation-coefficient & 0.066 & 0.061 & 0.101 & 0.444 & 0.117 \\
\cline { 2 - 7 } & p value & 0.531 & 0.567 & 0.345 & $<0.001^{* * *}$ & 0.272 \\
\hline \multirow{2}{*}{ CIC } & Correlation-coefficient & -0.396 & -0.457 & 0.484 & 0.256 & \\
\cline { 2 - 7 } & p value & $<0.001 * * *$ & $<0.001^{* * *}$ & $<0.001^{* * *}$ & $0.015^{*}$ & \\
\hline
\end{tabular}

Table 4. Correlation of individual biomarkers *p $<0.05, * * \mathrm{p}<0.01, * * * \mathrm{p}<0.001$.

\begin{tabular}{|l|l|l|l|l|l|}
\hline Biomarker models & Sensitivity & Specificity & OR & $\mathbf{9 5 \% C I}$ & $\boldsymbol{p}$-value \\
\hline Low complement $(\mathrm{C} 3<900 \mu \mathrm{g} / \mathrm{ml}$ or $\mathrm{C} 4<90 \mu \mathrm{g} / \mathrm{ml})$ & 51.8 & 65.1 & 2.01 & $(0.80-5.01)$ & 0.136 \\
\hline anti-dsDNA $(>100 \mathrm{IU} / \mathrm{ml})$ & 51.8 & 63.5 & 1.87 & $(0.75-4.67)$ & 0.178 \\
\hline Low complement or anti-dsDNA* & 74.1 & 52.4 & 3.14 & $(1.16-8.48)$ & 0.024 \\
\hline IL-6 $(>4.35 \mathrm{pg} / \mathrm{ml})^{* *}$ & 70.4 & 61.9 & 3.86 & $(1.46-10.18)$ & 0.006 \\
\hline CIC $(>4 \mathrm{RU} / \mathrm{ml}) * *$ & 70.4 & 66.7 & 4 & $(1.54-10.41)$ & 0.004 \\
\hline IL-6 or CIC** & 92.6 & 47.6 & 7.27 & $(1.99-26.63)$ & 0.003 \\
\hline Low complement, anti-dsDNA, IL-6, or CIC* & 96.3 & 28.6 & 5 & $(1.07-23.33)$ & 0.041 \\
\hline ESR $(>20 \mathrm{~mm} / \mathrm{hr})$ & 77.78 & 42.86 & 2.63 & $(0.93-7.39)$ & 0.068 \\
\hline ESR or CIC & 88.89 & 30.16 & 3.45 & $(0.93-12.87)$ & 0.065 \\
\hline Low complement, anti-dsDNA, ESR & 88.89 & 19.05 & 1.88 & $(0.49-7.30)$ & 0.36 \\
\hline Low complement, anti-dsDNA, ESR, CIC & 88.89 & 15.87 & 1.51 & $(0.38-5.98)$ & 0.558 \\
\hline
\end{tabular}

Table 5. Sensitivity and specificity of biomarker models in the active clinical SLEDAI. ${ }^{*} \mathrm{p}<0.05,{ }^{*} \mathrm{p}<0.01$.

a

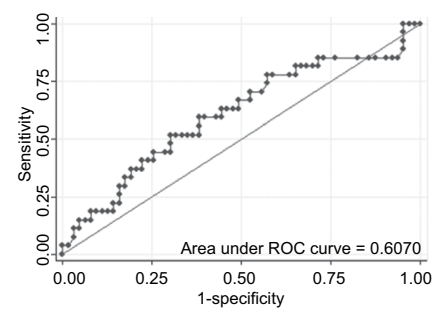

d

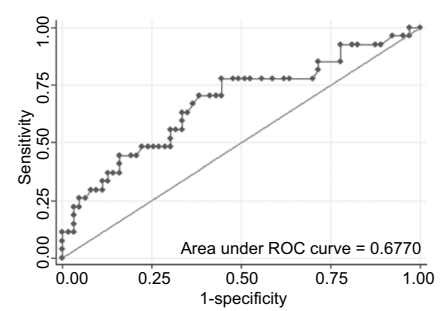

b

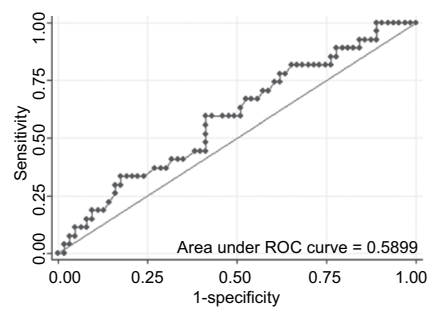

e

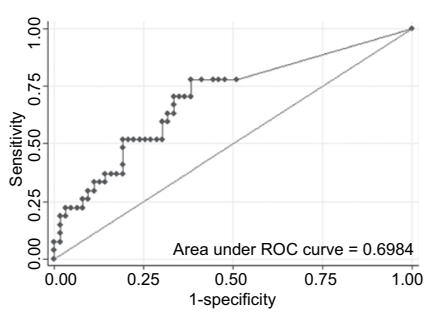

C

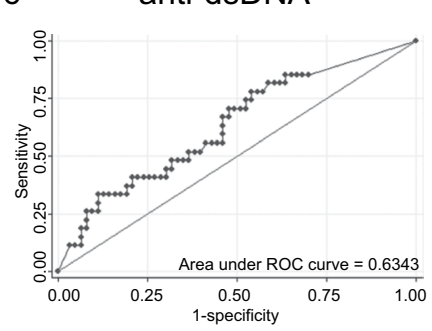

$f$

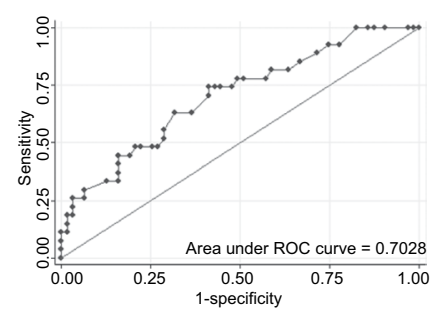

Figure 1. Receiver operating characteristic (ROC) curve and area under ROC curve of the following biomarkers; (a) Complement 3 (C3); (b) Complement 4 (C4); (c) anti-dsDNA; (d) Interleukin-6 (IL-6); (e) Circulating immune complex (CIC); (f) Erythrocyte sedimentation rate (ESR).

\section{Discussion}

The current biomarkers ( $\mathrm{C} 3, \mathrm{C} 4$, and anti-dsDNA) routinely used in clinical practice do not always correlate with the clinical manifestations of SLE patients. Complement can be changed by various factors and may not represent active clinical SLE ${ }^{20}$. The discovery of biomarkers for lupus nephritis has been extensively studied in order to identify better markers to predict disease flare ${ }^{21-23}$. The data of biomarkers for active non-renal SLE is quite limited, with minimal validation. Thirty percent of the patients in this cross-sectional study were clinically active, and forty-four percent of this active group were active in renal manifestation. The active SLE patients showed a significant difference of urine protein creatinine ratio compared to inactive group $(\mathrm{p}<0.001)$ but complement and anti-dsDNA did not differ between the two groups. Surprisingly, there was no significant correlation between C3 and C4 levels with clinical SLEDAI in this study.

IL-6 plays a critical role in the B cell hyperactivity and immunopathology of human SLE and may have a direct role in mediating tissue damage ${ }^{24}$. However, human SLE data show various results. Serum levels of IL-6 are elevated in human SLE and have correlated with disease activity ${ }^{9-11,25}$, while another study reports the increase 


\begin{tabular}{|c|c|c|c|c|c|c|c|}
\hline Clinical symptoms & ID-1 & ID-2 & ID-3 & ID-4 & ID-5 & ID-6 & ID-7 \\
\hline Seizure & - & - & - & - & - & - & - \\
\hline Psychosis & - & - & - & - & - & + & - \\
\hline Organic brain syndrome & - & - & - & - & - & - & - \\
\hline Visual disturbance & - & - & - & - & - & - & - \\
\hline Cranial nerve disorder & - & - & - & - & - & - & - \\
\hline Lupus headache & - & - & - & - & - & - & - \\
\hline CVA & - & - & - & - & - & - & - \\
\hline Vasculitis & - & - & - & - & - & - & - \\
\hline Arthritis & - & - & - & - & - & - & - \\
\hline Myositis & - & - & - & - & - & - & - \\
\hline Urinary casts & - & - & - & - & - & - & - \\
\hline Hematuria & - & - & - & + & - & - & - \\
\hline Proteinuria & - & + & + & - & - & - & + \\
\hline Pyuria & - & - & - & - & - & - & - \\
\hline Rash & + & - & - & - & - & - & - \\
\hline Alopecia & - & - & + & - & + & - & - \\
\hline Mucosal ulcers & - & - & - & - & - & - & - \\
\hline Pleurisy & - & - & - & - & - & - & - \\
\hline Pericarditis & - & - & - & - & - & - & - \\
\hline Low complement & - & - & - & - & - & - & - \\
\hline Increased DNA binding & - & - & - & - & - & -- & - \\
\hline Fever & - & - & - & - & - & - & - \\
\hline Thrombocytopenia & - & + & - & - & - & - & - \\
\hline Leukopenia & - & - & - & - & - & - & - \\
\hline SLEDAI-2K score & 2 & 5 & 6 & 4 & 2 & 8 & 4 \\
\hline Increased IL-6 & + & + & + & - & - & + & - \\
\hline Increased CIC & + & + & + & + & + & - & - \\
\hline Other clinical SLE & - & AIHA, Lymphopenia & $\begin{array}{l}\text { AIHA, Transverse } \\
\text { myelitis, Lymphopenia }\end{array}$ & Lymphopenia & - & Lymphopenia & - \\
\hline
\end{tabular}

Table 6. Clinical features and SLEDAI-2K of clinically active SLE patients with normal complement and antidsDNA.

of serum levels of IL-6 in SLE with active hematological disease, but did not correlate with other organ involvement evaluated by the BILAG index ${ }^{14}$. Here we demonstrated the serum levels of CIC and IL- 6 in the active SLE patients were significantly higher than those in the inactive SLE patients. Interestingly, we found the levels of IL-6 showed significant statistical correlation with clinical SLEDAI or modified SLEDAI-2K, but not with SLEDAI-2K, whereas CIC and anti-dsDNA correlated with both clinical SLEDAI or modified SLEDAI-2K. The reasons for this divergence could derive from the fact that IL- 6 did not correlate with complement and anti-dsDNA criteria that are included in SLEDAI-2K.

The presence of DNA-anti-DNA immune complexes correlates with SLE disease activity, and deposits of these complexes have been demonstrated in affected tissues ${ }^{26}$. Patients with lupus nephritis were reported to have a higher level of circulating soluble immune complexes than the ones without renal involvement; however, the study was conducted with only twenty-one patients ${ }^{27}$. Serum levels of CIC, which contain a fragment of complement C1q, were tested in these SLE patients and showed correlation with clinical SLEDAI and SLEDAI-2K which represented overall SLE disease activity (not just renal manifestation).

ESR is associated with disease activity in SLE measured by the SELENA-SLEDAI ${ }^{28}$. A change in ESR between two visits was correlated with a change in disease activities; however the change did not reach statistical significance ${ }^{28}$. Such a result is similar to our findings that ESR was significantly higher in active SLE and was correlated with disease activity, but the OR for predicting disease flare was insignificant.

ROC curve analysis suggested that serum levels of ESR, CIC and IL-6 were satisfactory discriminators for determining active or inactive SLE (based on clinical SLEDAI score). Of interest, our study showed that ESR presented the highest AUC in discriminating active SLE. These data suggested that ESR, CIC and IL-6 may be used as alternative biomarkers for determining SLE activity. A correlation between ESR and IL- 6 has been reported ${ }^{29}$. It will be more practical in the clinical setting if ESR can be used as an alternative biomarker instead of IL-6. However, the analysis showed that IL-6 gave a significant OR for predicting disease activity while ESR did not.

Pathogenesis of lupus disease is complex, and many mechanisms can contribute to the same phenotypes. The fact that CIC did not correlate with IL-6 but both markers did correlate with clinical activity suggested that a single biomarker may not be the ideal way to predict SLE disease activity. Next, we tried to analyze the biomarker models that could be the best predictor for disease flare. We selected the optimal cut-off point from the ROC curve of $\mathrm{CIC}$ as $4 \mathrm{RU} / \mathrm{ml}$, which gives a sensitivity of $70 \%$ and specificity of $67 \%$ respectively, whereas the IL-6 
cut-off is $4.35 \mathrm{pg} / \mathrm{ml}$, which gives a sensitivity of $70 \%$ and specificity of $62 \%$ respectively. The cut-off points of ESR $(>20 \mathrm{~mm} / \mathrm{hr})$, anti-dsDNA $(>100 \mathrm{IU}), \mathrm{C} 3(<900 \mu \mathrm{g} / \mathrm{ml})$, and C4 $(<90 \mu \mathrm{g} / \mathrm{ml})$ were the levels used in routine practice. If the threshold was adjusted, the OR may increase and provide better prediction for disease flare. We compared the sensitivity, specificity and odd ratios of combinations of individual markers. Models of either IL-6 or CIC showed the highest odd ratios for predict disease flare.

According to the SLE pathogenesis which involves both immune complex-mediated tissue injury and production of inflammatory cytokines (which may not only be limited to IL-6), the biomarkers that represent both processes should be used together to augment sensitivity in identifying disease flare. This idea was confirmed with the result that the combination of all serum biomarkers granted the highest sensitivity in detecting active clinical disease. The more markers used in the model, the higher was the sensitivity seen; however, the specificity was reduced.

Up to $70 \%$ of the patients in this study were clinically inactive, and some of them showed a rise of CIC and IL-6. We do not know whether those elevated levels happened before a disease flare or occurred because of a non-specific SLE condition such as non-obvious infection. A longitudinal study of SLE patients and controls from the non-autoimmune condition will provide more evidence to precisely determine the specificity of CIC and IL- 6 in the prediction of SLE flare. Also, the twenty-five percent of patients (7/27) with active clinical SLE showed a normal level of complement and anti-dsDNA. These patients showed clinical active non-renal manifestations (such as rash, lymphopenia, AIHA, psychosis, and myelitis) and the elevation of either CIC or IL-6.

In conclusion, the heterogeneity of SLE pathogenesis is the crucial factor that impacts various clinical phenotypes which subsequently influence the types of biomarkers to use for monitoring disease activity and the selection of specific treatments that suit individual patients. A combination of biomarkers involved in immune-complex mediated processes (such as CIC) and cytokine-mediated inflammation (such as IL-6) can increase the chance to predict SLE disease activity. We propose that a biomarker model of CIC and IL-6, based on the highest odds ratio, may be suitable for routine use as additional markers to monitor SLE disease activity.

\section{Methods}

Study population and data collection. Ninety SLE patients who followed up at Ramathibodi Hospital, Bangkok, Thailand during 2014-2015 were enrolled. All of the patients were older than 18 years and met 1997 ACR criteria ${ }^{30}$ or SLICC criteria $2012^{31}$ for the classification of SLE. The collected data were demographic information, disease manifestations, laboratory tests and history of treatment. The exclusion criteria were SLE patients with overlapping syndromes, active infection, cancer or previous history of cancer, and allergic disease. The study complied with the Declaration of Helsinki which was used by the Faculty of Medicine at Ramathibodi ethics committee in approving the research protocol. All patients (or their legally authorized representative) gave written informed consent.

Measurement of SLE disease activity. The Systemic Lupus Erythematosus Disease Activity Index (SLEDAI) is a global disease activity index that is widely used to monitor SLE disease activity ${ }^{32}$. Several versions of SLEDAI have been modified to make it more practical to use on a daily basis in clinical practice. Modification of SLEDAI (SLEDAI-2K) highly correlates with SLEDAI and can be used in clinical studies in SLE ${ }^{32}$. The modified SLEDAI-2K shows a good correlation with SLEDAI-2K ${ }^{33}$. Physicians evaluated the SLEDAI-2K in each SLE patient visiting the lupus clinic. The modified SLEDAI-2K, which did not include the low complement and the rise of anti-dsDNA, were analyzed as well. Active disease was defined if the Clinical SLEDAI score or modified SLEDAI-2K $\geq 1$, and inactive disease if the Clinical SLEDAI score or modified SLEDAI-2K $=0$.

Measurement of anti-dsDNA, interleukin-6 (IL-6) and circulating immune complex (CIC) levels. Anti-dsDNA levels were measured using an enzyme-linked immunosorbent assay (ELISA) method, following the manufacturer's recommended protocol (Euroimmun, Luebeck, Germany). Levels of IL-6 in the serum of SLE patients were measured using an electrochemiluminescence immunoassay (ECLIA) method (Roche Diagnostics GmbH, Mannheim, Germany). Circulating immune complex (CIC) levels were measured using an enzyme-linked immunosorbent assay (ELISA) method which provides a quantitative in vitro assay for human C1q-binding circulating immune complexes containing IgG antibodies (Euroimmun, Luebeck, Germany).

Statistical analysis. Statistical analysis was performed using PASW Statistics 18 computer software. The mean \pm SD was used to describe normally distributed data, median and IQR for skewed data, and proportion (\%) was used to describe categorical data. Student's $t$-test and Chi-square test/ Fisher's exact test were used for evaluating continuous and categorical data, respectively. Spearman's rank correlation was used to examine the correlation coefficient between parameters. Receiver operating characteristic (ROC) curves of ESR, IL-6, CIC, anti-dsDNA, C3, and C4 discriminated between active and inactive SLE. The cut-off of IL- 6 and CIC that yielded the best sensitivity and specificity were determined from the ROC curve. The sensitivity and specificity of ESR, anti-dsDNA, C3, and C4 were analyzed using the standard cut-off levels routinely used in clinical practice. Logistic regression analyses were performed to predict clinically active SLE. The results were considered as statistically significant if the $p$-value was $<0.05$.

Data Availability. The datasets generated during and/or analysed during the current study are not publicly available due to the privacy protection for patients but are available from the corresponding author on reasonable request. 


\section{References}

1. Rahman, A. \& Isenberg, D. A. Systemic lupus erythematosus. N Engl J Med 358, 929-939, https://doi.org/10.1056/NEJMra071297 (2008).

2. Herrmann, M., Voll, R. E. \& Kalden, J. R. Etiopathogenesis of systemic lupus erythematosus. Immunol Today 21, 424-426 (2000).

3. Jacob, N. \& Stohl, W. Cytokine disturbances in systemic lupus erythematosus. Arthritis Res Ther 13, 228, https://doi.org/10.1186/ ar3349 (2011).

4. Petri, M. Hopkins Lupus Cohort. 1999 update. Rheum Dis Clin North Am 26, 199-213, v (2000).

5. Furie, R., Toder, K. \& Zapantis, E. Lessons Learned From the Clinical Trials of Novel Biologics and Small Molecules in Lupus Nephritis. Semin Nephrol 35, 509-520, https://doi.org/10.1016/j.semnephrol.2015.08.012 (2015).

6. Luo, S., Wang, Y., Zhao, M. \& Lu, Q. The important roles of type I interferon and interferon-inducible genes in systemic lupus erythematosus. Int Immunopharmacol 40, 542-549, https://doi.org/10.1016/j.intimp.2016.10.012 (2016).

7. Apostolidis, S. A., Lieberman, L. A., Kis-Toth, K., Crispin, J. C. \& Tsokos, G. C. The dysregulation of cytokine networks in systemic lupus erythematosus. J Interferon Cytokine Res 31, 769-779, https://doi.org/10.1089/jir.2011.0029 (2011).

8. Hunter, C. A. \& Jones, S. A. IL-6 as a keystone cytokine in health and disease. Nat Immunol 16, 448-457, https://doi.org/10.1038/ ni.3153 (2015).

9. Grondal, G. et al. Cytokine production, serum levels and disease activity in systemic lupus erythematosus. Clin Exp Rheumatol 18, 565-570 (2000).

10. Linker-Israeli, M. et al. Elevated levels of endogenous IL-6 in systemic lupus erythematosus. A putative role in pathogenesis. $J$ Immunol 147, 117-123 (1991).

11. Peterson, E., Robertson, A. D. \& Emlen, W. Serum and urinary interleukin-6 in systemic lupus erythematosus. Lupus 5, 571-575, https://doi.org/10.1177/096120339600500603 (1996).

12. Swaak, A. J., van den Brink, H. G. \& Aarden, L. A. Cytokine production (IL-6 and TNF alpha) in whole blood cell cultures of patients with systemic lupus erythematosus. Scand J Rheumatol 25, 233-238 (1996).

13. Iwano, M. et al. Urinary levels of IL-6 in patients with active lupus nephritis. Clin Nephrol 40, 16-21 (1993).

14. Ripley, B. J., Goncalves, B., Isenberg, D. A., Latchman, D. S. \& Rahman, A. Raised levels of interleukin 6 in systemic lupus erythematosus correlate with anaemia. Ann Rheum Dis 64, 849-853, https://doi.org/10.1136/ard.2004.022681 (2005).

15. Levinsky, R. J., Cameron, J. S. \& Soothill, J. F. Serum immune complexes and disease activity in lupus nephritis. Lancet 1, 564-567 (1977).

16. Levinsky, R. J. Role of circulating soluble immune complexes in disease. Arch Dis Child 53, 96-99 (1978).

17. Swaak, A. J., Groenwold, J., Hannema, A. \& Hack, C. E. Correlation of disease activity with circulating immune complexes (C1qbA) and complement breakdown products (C3D) in patients with systemic lupus erythematosus. A prospective study. Rheumatol Int 5, 215-220 (1985).

18. Nydegger, U. E. \& Davis, J. St Soluble immune complexes in human disease. CRC Crit Rev Clin Lab Sci 12, 123-170 (1980).

19. Abrass, C. K., Nies, K. M., Louie, J. S., Border, W. A. \& Glassock, R. J. Correlation and predictive accuracy of circulating immune complexes with disease activity in patients with systemic lupus erythematosus. Arthritis Rheum 23, 273-282 (1980).

20. Walport, M. J. Complement and systemic lupus erythematosus. Arthritis Res 4(Suppl 3), S279-293, https://doi.org/10.1186/ar586 (2002).

21. Arriens, C., Wren, J. D., Munroe, M. E. \& Mohan, C. Systemic lupus erythematosus biomarkers: the challenging quest. Rheumatology (Oxford) 56, i32-i45, https://doi.org/10.1093/rheumatology/kew407 (2017).

22. Birmingham, D. J. et al. The complex nature of serum C3 and C4 as biomarkers of lupus renal flare. Lupus 19, 1272-1280, https:// doi.org/10.1177/0961203310371154 (2010).

23. Liu, C. C., Kao, A. H., Manzi, S. \& Ahearn, J. M. Biomarkers in systemic lupus erythematosus: challenges and prospects for the future. Ther Adv Musculoskelet Dis 5, 210-233, https://doi.org/10.1177/1759720X13485503 (2013).

24. Tackey, E., Lipsky, P. E. \& Illei, G. G. Rationale for interleukin-6 blockade in systemic lupus erythematosus. Lupus 13, 339-343, https://doi.org/10.1191/0961203304lu1023oa (2004).

25. Lu, R. et al. Dysregulation of innate and adaptive serum mediators precedes systemic lupus erythematosus classification and improves prognostic accuracy of autoantibodies. J Autoimmun 74, 182-193, https://doi.org/10.1016/j.jaut.2016.06.001 (2016).

26. Tan, E. M., Schur, P. H., Carr, R. I. \& Kunkel, H. G. Deoxybonucleic acid (DNA) and antibodies to DNA in the serum of patients with systemic lupus erythematosus. J Clin Invest 45, 1732-1740, https://doi.org/10.1172/JCI105479 (1966).

27. Cano, P. O., Jerry, L. M., Sladowski, J. P. \& Osterland, C. K. Circulating immune complexes in systemic lupus erythematosus. Clin Exp Immunol 29, 197-204 (1977).

28. Stojan, G., Fang, H., Magder, L. \& Petri, M. Erythrocyte sedimentation rate is a predictor of renal and overall SLE disease activity. Lupus 22, 827-834, https://doi.org/10.1177/0961203313492578 (2013).

29. Boss, B. \& Neeck, G. Correlation of IL-6 with the classical humoral disease activity parameters ESR and CRP and with serum cortisol, reflecting the activity of the HPA axis in active rheumatoid arthritis. $Z$ Rheumatol 59(Suppl 2), II/62-64 (2000).

30. Hochberg, M. C. Updating the American College of Rheumatology revised criteria for the classification of systemic lupus erythematosus. Arthritis Rheum 40, 1725, https://doi.org/10.1002/1529-0131(199709)40:9<1725::AID-ART29>3.0.CO;2-Y (1997).

31. Petri, M. et al. Derivation and validation of the Systemic Lupus International Collaborating Clinics classification criteria for systemic lupus erythematosus. Arthritis Rheum 64, 2677-2686, https://doi.org/10.1002/art.34473 (2012).

32. Gladman, D. D., Ibanez, D. \& Urowitz, M. B. Systemic lupus erythematosus disease activity index 2000. J Rheumatol 29, 288-291 (2002).

33. Uribe, A. G. et al. The Systemic Lupus Activity Measure-revised, the Mexican Systemic Lupus Erythematosus Disease Activity Index (SLEDAI), and a modified SLEDAI-2K are adequate instruments to measure disease activity in systemic lupus erythematosus. $J$ Rheumatol 31, 1934-1940 (2004).

\section{Acknowledgements}

We thank Euroimmun (Luebeck, Germany) for providing the reagents for testing human C1q-binding circulating immune complexes containing IgG antibodies. This research was supported by National Research Council of Thailand.

\section{Author Contributions}

"C.T., K.H. and P.P. wrote the main manuscript text and table. P.N. prepared Figure 1. C.S. and N.S. performed the laboratory testing. C.T., P.N., K.H. and P.P. analyzed the data. All authors reviewed the manuscript."

\section{Additional Information \\ Supplementary information accompanies this paper at https://doi.org/10.1038/s41598-018-20947-4.}

Competing Interests: PP is currently receiving grants from the Thailand Research Fund (\#RSA5980023 and IRN), National Research Council of Thailand and Ramathibodi Research Development Fund. Kenneth Hodge is supported by Rachadaphiseksompot Fund for Postdoctoral Fellowship, Chulalongkorn University. The remaining authors declared no conflicts of interest. 
Publisher's note: Springer Nature remains neutral with regard to jurisdictional claims in published maps and institutional affiliations.

(c) (i) Open Access This article is licensed under a Creative Commons Attribution 4.0 International License, which permits use, sharing, adaptation, distribution and reproduction in any medium or format, as long as you give appropriate credit to the original author(s) and the source, provide a link to the Creative Commons license, and indicate if changes were made. The images or other third party material in this article are included in the article's Creative Commons license, unless indicated otherwise in a credit line to the material. If material is not included in the article's Creative Commons license and your intended use is not permitted by statutory regulation or exceeds the permitted use, you will need to obtain permission directly from the copyright holder. To view a copy of this license, visit http://creativecommons.org/licenses/by/4.0/.

(C) The Author(s) 2018 